\title{
Role of Ischemic Preconditioning in the Cardioprotective Mechanisms of Monomeric C-Reactive Protein-Deposited Myocardium in a Rat Model
}

\author{
Eun Na Kim, M.D., Ph.D. ', Jae-Sung Choi, M.D., Ph.D. ${ }^{2}$, Chong Jai Kim, M.D., Ph.D. ', So Ra Kim, M.S. ${ }^{3}$, Se Jin Oh, M.D., Ph.D. ${ }^{2}$ \\ 'Department of Pathology, Asan Medical Center, University of Ulsan College of Medicine; ${ }^{2}$ Department of Thoracic and Cardiovascular Surgery, SMG-SNU \\ Boramae Medical Center, Seoul National University College of Medicine; ${ }^{3}$ Asan Laboratory of Perinatal Science, Asan Medical Center, University of Ulsan College of \\ Medicine, Seoul, Korea
}

\section{ARTICLE INFO}

Received July 15, 2020

Revised October 6, 2020

Accepted October 8, 2020

Corresponding author

Se Jin Oh

Tel $82-2-870-2295$

Fax 82-2-831-0714

E-mail wpwnn@snu.ac.kr

ORCID

https://orcid.org/0000-0002-1516-5313
Background: The deposition of monomeric C-reactive protein (mCRP) in the myocardium aggravates ischemia-reperfusion injury (IRI) and myocardial infarction. Ischemic preconditioning (IPC) is known to protect the myocardium against IRI.

Methods: We evaluated the effects of IPC on myocardium upon which mCRP had been deposited due to IRI in a rat model. Myocardial IRI was induced via ligation of the coronary artery. Direct IPC was applied prior to IRI using multiple short direct occlusions of the coronary artery. CRP was infused intravenously after IRI. The study included sham ( $n=3)$, IRI-only $(n=5),|R|+C R P(n=9)$, and IPC+|RI+CRP $(n=6)$ groups. The infarcted area and the area at risk were assessed using Evans blue and 2,3,5-triphenyltetrazolium staining. Additionally, mCRP immunostaining and interleukin-6 (IL-6) mRNA reverse transcription-polymerase chain reaction were performed.

Results: In the IRI+CRP group, the infarcted area and the area of mCRP deposition were greater, and the level of IL-6 mRNA expression was higher, than in the IRI-only group. However, in the IPC+IRI+CRP group relative to the IRI+CRP group, the relative areas of infarction ( $20 \%$ vs. $34 \%$, respectively; $p=0.079$ ) and mCRP myocardial deposition ( $21 \%$ vs. $44 \%$, respectively; $p=0.026$ ) were lower and IL-6 mRNA expression was higher (fold change: 407 vs. 326, respectively; $p=0.376$ ), although the difference in IL-6 mRNA expression was not statistically significant.

Conclusion: IPC was associated with significantly decreased deposition of mCRP and with increased expression of IL-6 in myocardium damaged by IRI. The net cardioprotective effect of decreased mCRP deposition and increased IL-6 levels should be clarified in a further study.

Keywords: Ischemic preconditioning, Reperfusion injury, C-reactive protein, Myocardial infarction

\section{Introduction}

Ischemic heart disease is one of the most common global causes of death, and is responsible for over 7.2 million deaths in the world annually [1]. In order to reduce the myocardial infarct size and prevent poor clinical outcomes, coronary artery reperfusion of the ischemic myocardium is performed using thrombolytic therapy, percutaneous coronary intervention, or coronary arterial bypass grafting. However, reperfusion of the ischemic myocardium can re- sult in paradoxical harmful effects that damage the myocardium; this is termed ischemic reperfusion injury (IRI) [2]. Approximately $50 \%$ of the final size of a myocardial infarction is due to IRI [1]. Roughly $10 \%$ of deaths and $25 \%$ of cases of cardiac failure following acute myocardial infarction can be attributed to IRI even after reperfusion of the ischemic heart [3]. Therefore, minimizing IRI is the most important strategy to salvage the myocardium after an ischemic event.

In 1986, Murry et al. [4] proposed the cardioprotective 
role of ischemic preconditioning (IPC); that is, multiple brief, non-lethal ischemic episodes followed by short reperfusion prior to the main prolonged ischemic injury reduces the infarct size due to the development of resistance to IRI and the inhibition of lethal reperfusion injury. For decades, researchers have studied the efficacy and mechanisms of IPC, and the myocardial protective effects of IPC have been demonstrated in animal studies [5] and in vivo human heart studies [6]. IPC not only reduces the infarct size by increasing the resistance of isolated myocytes to hypoxic injury [7], but also reduces anginal pain, ST segment elevation, and lactate production [8] and decreases the risk of post-ischemic arrhythmia [9]. IPC also slows metabolism and aids in the recovery of cardiac function after an ischemic event [10].

IPC-induced cardioprotective effects are known to be exerted by various triggers, receptors, and mediators. In particular, adenosine, opioid receptors [11], bradykinin [12], and free radicals [13] play important roles in IPC. In a heart preconditioned with IPC, lactate accumulates slowly, resulting in anti-infarct effects [10]. However, although numerous studies have been conducted over a long time period, the comprehensive mechanism of IPC is still controversial, and much remains unclear [4].

Emerging experimental evidence indicates that the deposition of monomeric C-reactive protein (mCRP) exacerbates the damage to the heart due to IRI $[14,15]$. C-reactive protein (CRP) is an acute-phase reactant protein mainly produced in the liver during systemic infections and inflammation. Not only is the serum level of CRP an important prognostic and predictive marker for various cardiovascular conditions following myocardial infarction, including clinical outcomes, death, and heart failure, but CRP itself also directly damages the cardiovascular tissue [16].

CRP in serum exists in a pentameric form (pCRP). When it encounters a damaged cell membrane, it undergoes structural changes from pCRP to mCRP [17]. Subsequently, mCRP is deposited in the damaged tissue, thereby activating reactive oxygen species [18] and the complement system [19]. This aggravates the inflammatory process and exacerbates myocardial damage [15]. Using a rat IRI model, we previously confirmed that if the serum CRP level is high during the myocardial ischemic-reperfusion insult, serum CRP is deposited in the myocardium as mCRP, and a larger myocardial infarction is observed [14]. Additionally, our previous work demonstrated that the microRNA profile of the myocardial area at risk (AAR) changed drastically when the CRP level was high during the isch- emic-reperfusion injury [20].

However, no studies have focused on the effects of IPC on ischemic and damaged myocardium upon which mCRP has been deposited. Therefore, in this study, we used a rat acute myocardial IRI model to investigate whether IPC is protective against mCRP-induced myocardial damage in situations involving IRI.

\section{Methods}

\section{Animals}

We set up a myocardial IRI model using female SpragueDawley rats weighing between 220 and $270 \mathrm{~g}$ with a gestational age of 10-14 weeks. The animals were treated according to the Guide for the Care and Use of Laboratory Animals (National Academy of Sciences, Washington, DC, USA). The protocols for animal use were approved by the Institutional Animal Care and Use Committee at the SMG-SNU Boramae Medical Center Biomedical Research Institute (approval no., 2016-0027). Anesthesia was administered in the form of the inhalation of isoflurane (4\%) for induction, followed by the intraperitoneal administration of tiletamine $\mathrm{HCl}$ and zolazepam $\mathrm{HCl}$ (Zoletil 50, $0.12 \mathrm{~mL}$; Virbac, Carros, France) and xylazine (Rompun, 2\%, 0.02 $\mathrm{mL}$; Bayer Healthcare, Loos, France) for maintenance.

The rats were intubated with $16 \mathrm{G}$ intravenous catheters (REF 382457; BD Medical, Sandy, UT, USA) and connected to rodent ventilators (Model 683; Harvard Apparatus, Holliston, MA, USA). Positive-pressure ventilation with room air at a tidal volume of $2.5 \mathrm{~mL}(10 \mathrm{~mL} / \mathrm{kg}$, 60 breaths $/ \mathrm{min})$ was used to prevent atelectasis during the procedure. We approached the heart through a left thoracotomy via the fourth intercostal space. The pericardium was opened to expose the left coronary artery. Myocardial ischemic injury was produced by ligating the left anterior descending coronary artery (LAD) approximately $2 \mathrm{~mm}$ distal to its origin using 6-0 nylon double sutures, buttressed with a small piece of plastic tubing (Fig. 1). After 45 minutes of ischemia, we loosened the sutures to allow reperfusion for 45 minutes. The pericardium was left open to expose the LAD during the procedure.

\section{Experimental protocols}

The experimental protocols are illustrated in Fig. 2. In the sham group $(n=3)$, thoracotomy and pericardiotomy were performed, and the pericardium was opened and maintained for 90 minutes without any manipulation. Sub- 
sequently, the rats were euthanized, and autopsy was performed (Fig. 2). In the group with IRI only (the IRI-only group, $n=5$ ), the myocardium was excised quickly after 45 minutes of LAD ligation and 45 minutes of reperfusion (Fig. 2). In the group treated with CRP following IRI (the IRI+CRP group, n=9), high-purity (>99\%) human CRP obtained from human plasma (C4063; Sigma-Aldrich, St. Louis, MO, USA) was infused via the femoral vein after 45 minutes of ischemia with LAD ligation, immediately prior to reperfusion (Fig. 2). In the group treated with IPC followed by IRI and CRP injection (the IPC+IRI+CRP group, $\mathrm{n}=6$ ), IPC was applied before LAD ligation; IPC included 3 occlusions for 3 minutes each with a 5 -minute period of reperfusion after each occlusion (Fig. 2).

\section{Evans blue and TTC staining to identify the infarcted area and the AAR}

To identify the non-ischemic area, the ischemic but not infarcted viable area (the AAR), and the infarcted area, we

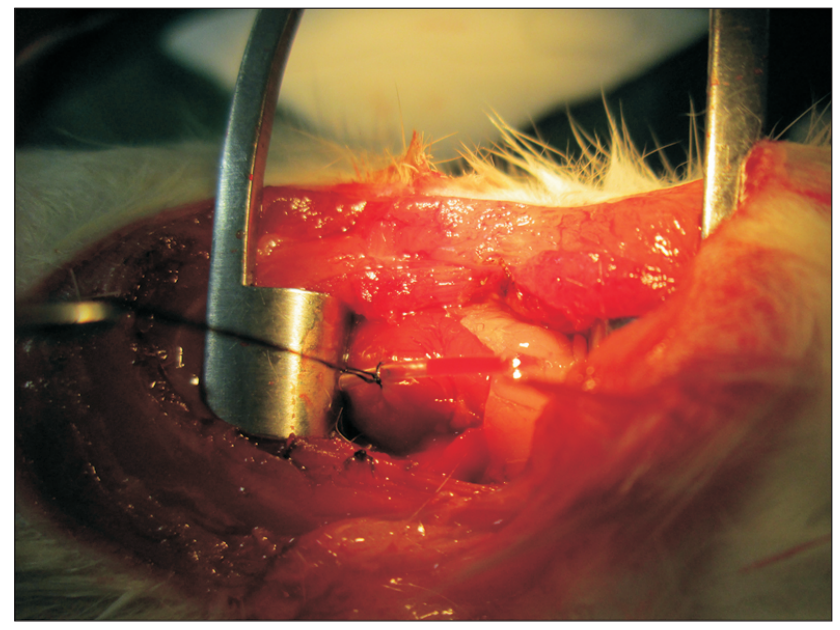

Fig. 1. Ligation of the left anterior descending coronary artery, buttressed with a plastic tube. performed staining with Evans blue and 2,3,5-triphenyltetrazolium chloride (TTC; Sigma-Aldrich). After euthanasia and before the removal of the heart, $1 \mathrm{~mL}$ of a diluted heparin solution $(2,500 \mathrm{IU}$ heparin/mL) was infused via the coronary ostia after the ascending aorta was clamped. We ligated the LAD artery using 6-0 nylon sutures, and we injected 1\% Evans blue solution to stain the perfused non-ischemic myocardium. Neither component of the ischemic area (i.e., neither the AAR nor the infarcted area) is stained by Evans blue solution. After perfusion with Evans blue, the heart was cut into 4 transverse sections at regular intervals from the apex to the base. One of the middle sections was used to measure the ischemic and infarcted regions. This mid-portion was sliced again in 4-mm slices, and 1 slice was incubated with TTC dissolved in 100 $\mathrm{mmol} / \mathrm{L}$ of phosphate buffer for 15 minutes. Under TTC staining, the viable area of the myocardium (i.e., the non-ischemic area) and the viable AAR are stained deep red, while the infarcted zone remains unstained and is therefore white. Thus, double staining with Evans blue and TTC stains the infracted area white, the AAR deep red, and the non-ischemic area blue.

\section{Histopathologic analysis and immunohistochemistry}

The middle section of the excised heart was fixed in $10 \%$ buffered formalin and embedded in paraffin. The $4-\mu \mathrm{m}$ tissue sections were stained using hematoxylin and eosin (H\&E) and immunostained with a human monoclonal anti-CRP antibody (C1688; Sigma-Aldrich; 1:400 dilution) that specifically detects the $24-\mathrm{kDa}$ monomeric CRP epitope. An OptiView DAB immunohistochemical detection kit (Roche Diagnostics, Mannheim, Germany) and a Benchmark XT autoimmunostainer (Ventana Medical Systems, Tucson, AZ, USA) were used for immunostaining. A thorough histopathologic examination with microscopy was performed by 1 pathologist (E.N.K.).

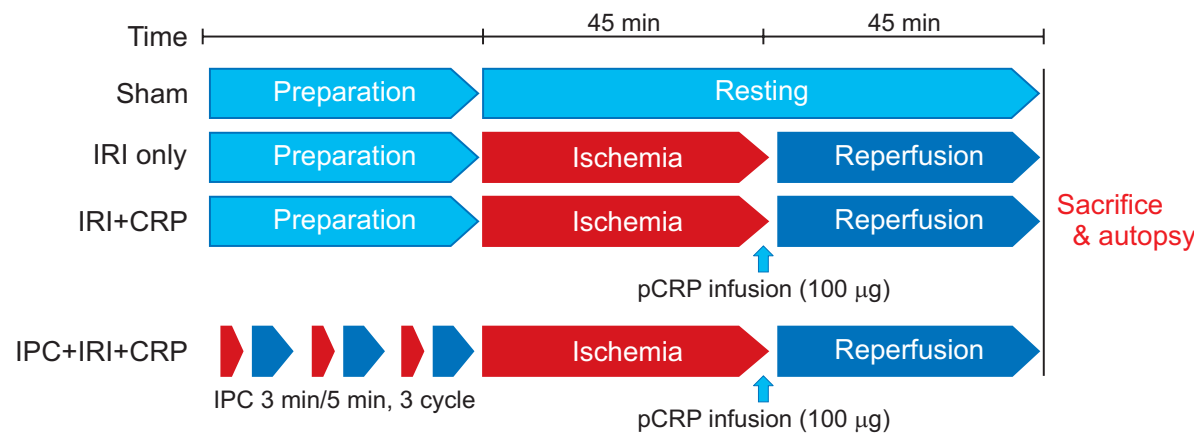

Fig. 2. Experimental protocols. IRI, ischemia-reperfusion injury; CRP, C-reactive protein; $\mathrm{pCRP}$, pentameric C-reactive protein; IPC, ischemic preconditioning. 


\section{Image analysis}

Images of the heart specimens stained with Evans blue and TTC were captured with a digital camera (DP26; Olympus, Tokyo, Japan). Digital images of mCRP immunostaining were acquired using the Vectra automated imaging system (PerkinElmer, Waltham, MA, USA). The areas of the infarcted myocardium (white zone) and the AAR (red zone) were automatically calculated using inForm (PerkinElmer) imaging analysis software. The size of the infarct was expressed as a percentage of the whole ischemic area [infarct/(infarct+AAR) $\times 100]$. mCRP immunolabeling was used to examine the tissue distribution within each section. mCRP immunopositivity was expressed as a percentage of the whole ischemic area [mCRP-immunostained area/(infarct+AAR) $\times 100]$, as previously reported [14].

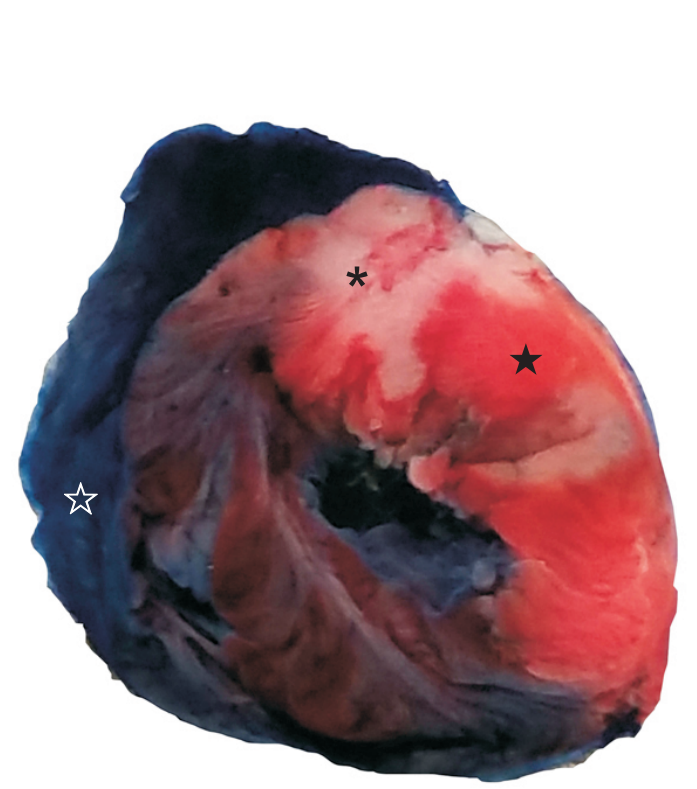

Fig. 3. Evans blue and 2,3,5-triphenyltetrazolium chloride staining. White area $\left(^{*}\right)$ : infarcted area. Red area $(\star)$ : area at risk (ischemic but not infarcted). Blue area (交): non-ischemic area.

\section{Analysis of IL-6 mRNA expression in rat myocardium}

RNA was prepared using a miRNeasy Mini Kit (Qiagen, Hilden, Germany) according to the manufacturer's instructions. The extracted RNA $(1 \mu \mathrm{g})$ was reverse transcribed using a reverse transcription system (Promega, Madison, WI, USA), and complementary DNA was amplified using the GeneAmp PCR System 9700 (Applied Biosystems, Foster City, CA, USA). Quantitative reverse transcriptase-polymerase chain reaction analysis of interleukin (IL)-6 was performed using TaqMan Gene Expression Assays (Rn01410330_m1; Applied Biosystems) and the 7900HT Fast Real-Time PCR System (Applied Biosystems). The rat ACTB (Rn00667869_m1; Applied Biosystems) endogenous control was used for normalization.

\section{Statistical analysis}

Data were expressed as the mean and standard deviation and plotted as the mean with the standard error of the mean. For comparisons between 2 groups, the MannWhitney U-test was used for continuous variables. A p-value $<0.05$ was considered to indicate statistical significance. Data analyses were performed using GraphPad Prism ver. 5.0 software (GraphPad Software, San Diego, CA, USA).

\section{Results}

\section{Areas of infarcted and ischemic myocardium}

After Evans blue and TTC staining, the non-ischemic area was stained blue, the AAR was stained red, and the infarcted area was stained white, as depicted in Fig. 3. As in our previous study [14], the areas of myocardium damaged by IRI demonstrated cellular changes along with contraction bands with intensely eosinophilic intracellular
A

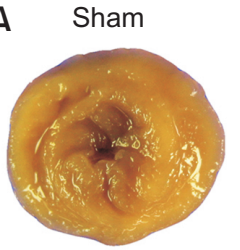

B

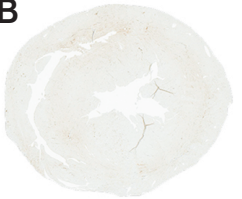

IRI only
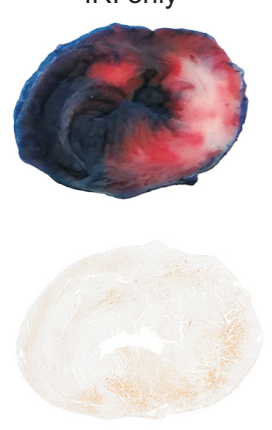

$\mathrm{IRI}+\mathrm{CRP}$
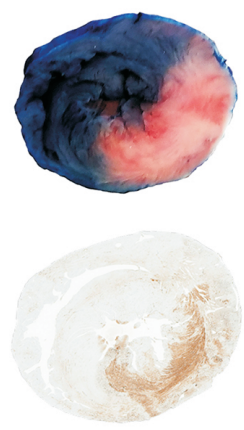

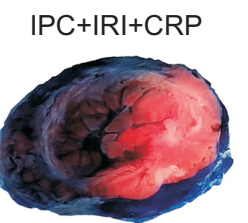

Fig. 4. (A) Sham group withoutstaining. TTC and Evans blue staining in the IRI-only, IRI+CRP, and IPC+IRI+CRP groups. (B) mCRP immunohistochemistry in the sham, IRI-only, $I R I+C R P$, and IPC+IRI+CRP groups. TTC, 2,3,5-triphenyltetrazolium chloride; IRI, ischemia-reperfusion injury; CRP, C-reactive protein; IPC, ischemic preconditioning; $\mathrm{mCRP}$, monomeric C-reactive protein. 


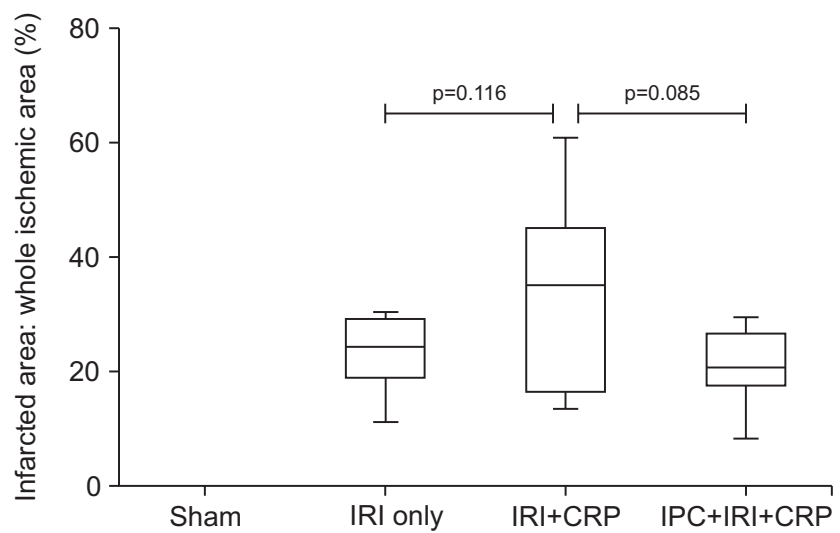

Fig. 5. Ratio of the infarcted area to the whole ischemic area. IRI, ischemia-reperfusion injury; CRP, C-reactive protein; IPC, ischemic preconditioning.

stripes on H\&E staining, thus confirming that IRI was properly induced in the experiment [21]. The ratio of the infarcted area to the whole ischemic area [infarct/(infarct+AAR $\times 100$ ] was higher in the IRI+CRP group than in the IRI-only group $(34 \% \pm 15 \%$ versus $23 \% \pm 7 \%$, respectively; $\mathrm{p}=0.079)$. The relative size of the infarcted area was lower in the IPC+IRI+CRP group than in the IRI+CRP group, although this difference was not statistically significant $(21 \% \pm 7 \%$ versus $34 \% \pm 15 \%$, respectively; $\mathrm{p}=0.079)$ (Figs. 4A, 5).

\section{mCRP immunohistochemistry}

While mCRP staining was faint and non-specific in the sham and IRI-only groups, floating serum CRP was strongly and diffusely deposited on the damaged myocardium in the IRI+CRP group, in the viable AAR as well as in the infarcted area. This finding is consistent with our previous results [14] (Fig. 4B). However, after applying direct IPC, the area of $\mathrm{mCRP}$ deposition in the ischemic myocardium relative to the whole ischemic area [mCRPimmunostained area/(infarct+AAR) $\times 100$ ] was significantly lower than in the IRI+CRP group $(21 \% \pm 16 \%$ versus $44 \% \pm$ $19 \%$, respectively; $\mathrm{p}=0.026$ ) (Figs. $4 \mathrm{~B}, 6$ ).

\section{IL-6 mRNA expression increased after CRP injection and IPC}

The level of IL- 6 mRNA expression was highest in the IPC+IRI+CRP group (fold change, 408273), followed by the IRI+CRP (fold change, 326 \pm 157 ) and IRI-only (fold change, $198 \pm 113)$ groups. However, these differences were not statistically significant (IRI-only versus IRI+CRP, $p=0.154$;

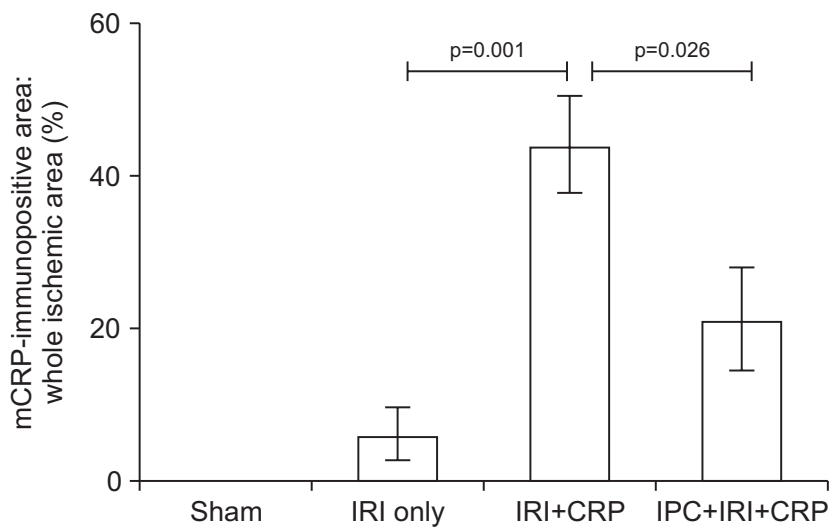

Fig. 6. Ratio of the mCRP-immunopositive area to the whole ischemic area. mCRP, monomeric C-reactive protein; IRI, ischemia-reperfusion injury; CRP, C-reactive protein; IPC, ischemic preconditioning.

\section{IL-6 mRNA expression}

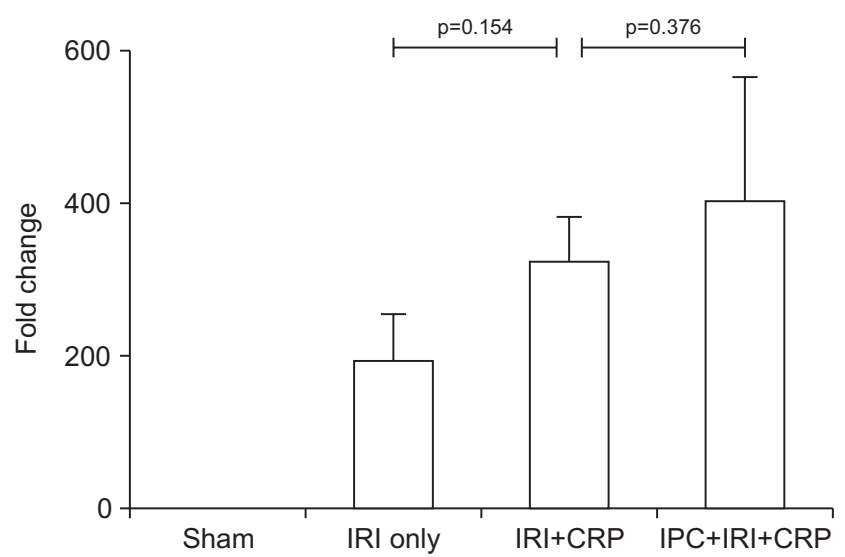

Fig. 7. IL-6 mRNA expression. IL-6, interleukin-6; mRNA, messenger RNA; IRI, ischemia-reperfusion injury; CRP, C-reactive protein; IPC, ischemic preconditioning.

IRI+CRP versus IPC+IRI+CRP, $\mathrm{p}=0.376)$ (Fig. 7).

\section{Discussion}

To our knowledge, this is the first report of the cardioprotective effect of IPC on mCRP-deposited ischemic myocardium. In this study, we found that short-term direct IPC prior to IRI and CRP infusion diminished mCRP deposition in the myocardium, reduced infarction size, and increased the expression of IL-6 mRNA.

Previous studies have documented that mCRP deposition aggravated IRI-induced myocardial infarction. Thiele et al. [15] reported that in a rat model of IRI, mCRP was localized to the infarcted myocardium and aggravated inflammation via the phospholipase A2-dependent dissocia- 
tion of circulating pCRP to mCRP. Pepys et al. [22] reported that they used 1,6-bis(phosphocholine)-hexane, which could bind and inhibit human CRP, to reduce myocardial infarction size. Additionally, we previously reported that mCRP was deposited not only in the infarcted area, but also in the AAR, in a process accompanied by mitochondrial damage and complement activation [14].

IL-6 is a pleiotropic pro-inflammatory cytokine and a main factor in the stimulation of acute-phase proteins, such as CRP [23]. IL-6 is known to be released from cardiomyocytes under hypoxic conditions, such as in myocytes in the border zone of a myocardial infarction, and IL-6 derived from hypoxic myocytes may play an important role in the aggravation of myocardial dysfunction following IRI [24]. Therefore, IL-6 was thought to result in hypertrophy and heart failure after ischemia by reducing the contractility of the myocardium [25]. However, many studies have demonstrated that higher IL- 6 levels during preconditioning play an organo-protective role [26-28]. Dawn et al. [26] showed that IPC markedly upregulated IL-6 expression in the ischemic/reperfused myocardium. Furthermore, they demonstrated that IL-6 signaling plays an obligatory role in late preconditioning because IL-6 is required for JAK-STAT (Janus kinases-signal transducer and activator of transcription) signaling and the upregulation of inducible nitric oxide (NO) synthase and cyclooxygenase-2, which are co-mediators of late preconditioning and thus can aid in cardioprotection [26]. Similarly, Waldow et al. [27] reported that when remote IPC was applied before IRI in a porcine lung, IL-6 increased more consistently than in trials involving IRI alone, and lung damage was alleviated. In their in vivo study using ventricular cardiomyocytes isolated from rat hearts, Smart et al. [28] demonstrated that IL-6 induced the PI-3 kinase- and NO-dependent protection of cardiomyocytes, which was associated with alterations in mitochondrial $\mathrm{Ca}^{2+}$ handling, inhibition of reperfusion-induced mitochondrial depolarization, swelling and loss of structural integrity, and suppression of cytosolic $\mathrm{Ca}^{2+}$ transients. Additionally, in the present study, we confirmed that administering IPC prior to myocardial damage by IRI and mCRP deposition increased IL- 6 mRNA expression and decreased the size of the myocardial infarct.

However, whether increased IL-6 secretion plays an organoprotective role is still a subject of debate, and no studies in the existing literature have described the net effect of IL-6. In a porcine lung IRI model, Harkin et al. [29] reported that IPC lowered IL- 6 levels and resulted in a lung-protective effect. The downstream pathways through which IL-6 plays a cardioprotective role are not well understood. Additionally, no detailed studies have been conducted to identify the specific signaling pathways involved when the IL-6 level increases after mCRP deposition. This study may be regarded as a pilot study demonstrating the beneficial effect of IL- 6 induced by IPC, and a further targeted study of IL- 6 is required to elucidate the mechanisms through which IL-6 acts on mCRP-deposited damaged tissue.

\section{Limitations}

In this study, some observations did not demonstrate statistical significance. However, when CRP was deposited, the infarction area increased, matching the trend reported in our previous work [14]. Animal studies with a larger sample size are required to further validate these findings. Additionally, a group administered IPC+IRI without CRP infusion should have been included in the study protocol to isolate the effect of CRP in the IPC model. In addition, a disparity may exist between the findings in animals and real-world clinical situations. Indeed, numerous attempts to prevent IRI that worked in animal experiments have been ineffective in clinical studies [1]. We used an ischemia model involving direct occlusion of the healthy coronary artery in young rats, which would be very invasive in the context of practical clinical applications. It is also virtually impossible to predict when profound ischemia will occur and apply preconditioning before IRI. Therefore, creating a useful clinical application from our experimental results may be difficult. Furthermore, to extend the animal model to reflect actual clinical situations, animal models including older animals and those with comorbidities such as diabetes, hyperlipidemia, atherosclerosis, and hypertension should be used [1].

Many clinical situations exist in which serum CRP can be elevated. Baseline CRP levels are moderately elevated in obese persons, those who smoke, and those with diabetes or hypertension. Additionally, CRP levels increase dramatically in patients with myocardial infarction [30]. If a patient with a cardiovascular event has an elevated serum CRP level for any reason, the serum CRP will degrade the myocardial function as it is deposited in the damaged myocardium. Therefore, we can infer that in these clinical situations, IPC will function to protect the mCRP-deposited myocardium. Additionally, our experiments provide clues to the mechanisms of aggravation of ischemia due to mCRP and the protective mechanisms of IPC. If the mechanism by which IPC protects myocardium that has under- 
gone mCRP deposition is fully understood, it will provide a basis for the development of a preconditioning mimetic agent.

\section{Conclusion}

Our results showed that IPC significantly decreased the deposition of mCRP in damaged myocardium and tended to increase the expression of IL- 6 . The net protective effect of decreased mCRP deposition and increased IL- 6 expression on myocardium damaged by IRI is still unclear and should be investigated in a further study.

\section{Conflict of interest}

No potential conflict of interest relevant to this article was reported.

\section{Acknowledgments}

We thank Kyung Min Park, Gil-Je Lee, and Yun Jae Kim for the image analysis and Eun Jung Jeon for the molecular work.

\section{Funding}

This work was funded by a clinical research grant-in-aid from the Seoul Metropolitan Government Seoul National University (SMG-SNU) Boramae Medical Center (03-201511). The funders had no role in the study design, data collection and analysis, decision to publish, or preparation of the manuscript.

\section{ORCID}

Eun Na Kim: https://orcid.org/0000-0003-2992-7881 Jae-Sung Choi: https://orcid.org/0000-0001-5408-9029 Chong Jai Kim: https://orcid.org/0000-0002-2844-9446 So Ra Kim: https://orcid.org/0000-0001-9032-0814 Se Jin Oh: https://orcid.org/0000-0002-1516-5313

\section{References}

1. Yellon DM, Hausenloy DJ. Myocardial reperfusion injury. N Engl J Med 2007;357:1121-35.

2. Jennings RB, Sommers HM, Smyth GA, Flack HA, Linn H. Myocardial necrosis induced by temporary occlusion of a coronary artery in the dog. Arch Pathol 1960;70:68-78.

3. Keeley EC, Boura JA, Grines CL. Primary angioplasty versus intra- venous thrombolytic therapy for acute myocardial infarction: a quantitative review of 23 randomised trials. Lancet 2003;361:13-20.

4. Murry CE, Jennings RB, Reimer KA. Preconditioning with ischemia: a delay of lethal cell injury in ischemic myocardium. Circulation 1986;74:1124-36.

5. Liu Y, Downey JM. Ischemic preconditioning protects against infarction in rat heart. Am J Physiol 1992;263:H1107-12.

6. Yellon DM, Alkhulaifi AM, Pugsley WB. Preconditioning the human myocardium. Lancet 1993;342:276-7.

7. Armstrong S, Ganote CE. Adenosine receptor specificity in preconditioning of isolated rabbit cardiomyocytes: evidence of $A 3$ receptor involvement. Cardiovasc Res 1994;28:1049-56.

8. Deutsch E, Berger M, Kussmaul WG, Hirshfeld JW Jr, Herrmann $\mathrm{HC}$, Laskey WK. Adaptation to ischemia during percutaneous transluminal coronary angioplasty: clinical, hemodynamic, and metabolic features. Circulation 1990;82:2044-51.

9. Wu ZK, Iivainen T, Pehkonen E, Laurikka J, Tarkka MR. Ischemic preconditioning suppresses ventricular tachyarrhythmias after myocardial revascularization. Circulation 2002;106:3091-6.

10. Murry CE, Richard VJ, Reimer KA, Jennings RB. Ischemic preconditioning slows energy metabolism and delays ultrastructural damage during a sustained ischemic episode. Circ Res 1990;66:913-31.

11. McCully JD, Uematsu M, Parker RA, Levitsky S. Adenosine-enhanced ischemic preconditioning provides enhanced cardioprotection in the aged heart. Ann Thorac Surg 1998;66:2037-43.

12. Wall TM, Sheehy R, Hartman JC. Role of bradykinin in myocardial preconditioning. J Pharmacol Exp Ther 1994;270:681-9.

13. Baines CP, Goto M, Downey JM. Oxygen radicals released during ischemic preconditioning contribute to cardioprotection in the rabbit myocardium. J Mol Cell Cardiol 1997;29:207-16.

14. Oh SJ, Na Kim E, Jai Kim C, Choi JS, Kim KB. The effect of C-reactive protein deposition on myocardium with ischaemia-reperfusion injury in rats. Interact Cardiovasc Thorac Surg 2017;25:260-7.

15. Thiele JR, Habersberger J, Braig D, et al. Dissociation of pentameric to monomeric $C$-reactive protein localizes and aggravates inflammation: in vivo proof of a powerful proinflammatory mechanism and a new anti-inflammatory strategy. Circulation 2014;130:35-50.

16. Li JJ, Fang CH. C-reactive protein is not only an inflammatory marker but also a direct cause of cardiovascular diseases. Med Hypotheses 2004;62:499-506.

17. Khreiss T, Jozsef L, Potempa LA, Filep JG. Loss of pentameric symmetry in $C$-reactive protein induces interleukin- 8 secretion through peroxynitrite signaling in human neutrophils. Circ Res 2005;97:6907.

18. Thiele JR, Zeller J, Kiefer J, et al. A conformational change in c-reactive protein enhances leukocyte recruitment and reactive oxygen species generation in ischemia/reperfusion injury. Front Immunol 2018;9:675.

19. Thompson D, Pepys MB, Wood SP. The physiological structure of 
human $C$-reactive protein and its complex with phosphocholine. Structure 1999;7:169-77.

20. Kim EN, Kim CJ, Kim SR, et al. High serum CRP influences myocardial miRNA profiles in ischemia-reperfusion injury of rat heart. PLoS One 2019;14:e216610.

21. Basso C, Thiene G. The pathophysiology of myocardial reperfusion: a pathologist's perspective. Heart 2006;92:1559-62.

22. Pepys MB, Hirschfield GM, Tennent GA, et al. Targeting C-reactive protein for the treatment of cardiovascular disease. Nature 2006;440: 1217-21.

23. Gauldie J, Richards C, Harnish D, Lansdorp P, Baumann H. Interferon beta 2/B-cell stimulatory factor type 2 shares identity with monocyte-derived hepatocyte-stimulating factor and regulates the major acute phase protein response in liver cells. Proc Natl Acad Sci U S A 1987;84:7251-5

24. Gwechenberger M, Mendoza LH, Youker KA, et al. Cardiac myocytes produce interleukin- 6 in culture and in viable border zone of reperfused infarctions. Circulation 1999;99:546-51.

25. Tsutamoto T, Hisanaga T, Wada A, et al. Interleukin- 6 spillover in the peripheral circulation increases with the severity of heart failure, and the high plasma level of interleukin-6 is an important prognostic predictor in patients with congestive heart failure. J Am Coll Cardiol 1998;31:391-8.

26. Dawn B, Xuan YT, Guo Y, et al. IL-6 plays an obligatory role in late preconditioning via JAK-STAT signaling and upregulation of iNOS and COX-2. Cardiovasc Res 2004;64:61-71.

27. Waldow T, Alexiou K, Witt W, et al. Protection against acute porcine lung ischemia/reperfusion injury by systemic preconditioning via hind limb ischemia. Transpl Int 2005;18:198-205.

28. Smart N, Mojet MH, Latchman DS, Marber MS, Duchen MR, Heads RJ. IL-6 induces PI 3-kinase and nitric oxide-dependent protection and preserves mitochondrial function in cardiomyocytes. Cardiovasc Res 2006;69:164-77.

29. Harkin DW, Barros D'Sa AA, McCallion K, Hoper M, Campbell FC. Ischemic preconditioning before lower limb ischemia: reperfusion protects against acute lung injury. J Vasc Surg 2002;35:1264-73.

30. Kitsis RN, Jialal I. Limiting myocardial damage during acute myocardial infarction by inhibiting $C$-reactive protein. N Engl J Med 2006;355:513-5 